\title{
CHILDREN ARE BOTH MORE CONSERVATIVE AND MORE LIKELY TO GENERALIZE THAN ADULTS FOR THE SAME REASON
}

\author{
ADELE GOLDBERG ${ }^{* 1}$ \\ *Corresponding Author: adele@princeton.edu \\ ${ }^{1}$ Psychology Department, Princeton University, New Jersey, USA
}

Children have been argued to be both more conservative than adults, initially appearing to be less productive with their linguistic constructions than adults; they have also been argued to be over-eager generalizers when compared with adults, insofar as they are more likely to regularize complex linguistic input. This raises an apparent paradox: how can children be more likely than adults to both under-generalize (be conservative) and over-generalize (regularize)? The answer lies in the fact that children are less adept at aligning exemplars within the high-dimensional conceptual space in which our long-term knowledge of language - our constructicon - is represented. Children are therefore more prone than adults to be conservative, when they fail to recognize relevant similarities among exemplars, and simultaneously more likely to oversimplify or generalize, when they fail to appreciate relevant distinctions. 